\title{
An inner disk below the ADAF: the intermediate spectral state of black hole accretion
}

\author{
B. F. Liu ${ }^{1}$, F. Meyer ${ }^{2}$, and E. Meyer-Hofmeister ${ }^{2}$ \\ 1 National Astronomical Observatories/Yunnan Observatory, Chinese Academy of Sciences, PO Box 110, Kunming 650011, \\ PR China \\ e-mail: bfliu@ynao.ac.cn \\ 2 Max-Planck-Institut für Astrophysik, Karl- Schwarzschildstr. 1, 85740 Garching, Germany \\ e-mail: [frm; emm]@mpa-garching .mpg.de
}

Received 13 April 2006 / Accepted 1 June 2006

\section{ABSTRACT}

\begin{abstract}
Aims. The hard and soft spectral states of black hole accretion are understood as connected with ADAF accretion (truncated disk) and standard disk accretion, respectively. However, observations indicate the existence of cool gas in the inner region at times when the disk is already truncated outside. We try to shed light on these not yet understood intermediate states.

Methods. The disk-corona model allows to understand the spectral state transitions as caused by changes of the mass flow rate in the disk and provides a picture for the accretion geometry when disk truncation starts at the time of the soft/hard transition, the formation of a gap in the disk filled by an advection-dominated flow (ADAF) at the distance where the evaporation is maximal. We study the interaction of such an ADAF with an inner thin disk below.

Results. We show that, when the accretion rate is not far below the transition rate, an inner disk could exist below an ADAF, leading to an intermediate state of black hole accretion.
\end{abstract}

Key words. accretion, accretion disks - black hole physics - X-rays: binaries

\section{Introduction}

It is now widely accepted that the hard and soft spectral states of black holes correspond to accretion in form of an advectiondominated flow (ADAF) and a thin disk, respectively (Esin et al. 1997). But observations also show an intermediate state which often appears in transitions between these two states.

The hard states are understood as a consequence of interaction of the corona and the underlying disk which yields evaporation of gas from the disk to the hot coronal flow, and leads to a truncation of the accretion disk at some distance (Meyer et al. $2000 \mathrm{~b}$ ). Inside this truncation radius there is only a hot, optically thin flow. The soft state, on the other hand, is explained as the optically thick, standard accretion disk extending down to the last stable orbit, revealing itself in the characteristic multitemperature blackbody spectrum.

But observations also show an intermediate state which appears often in transitions between these two states (McClintock \& Remillard 2006). After the soft/hard transition the spectra are not always solely produced by an optically thin accretion flow, the occurrence of reflection and a $\mathrm{Fe} \mathrm{K} \alpha$ line indicate cool matter in the inner region (Życki et al. 1998). These intermediate states often persist for times significantly longer than the viscous timescale of the disk and can thus not be explained only by the fading or build-up of a disk during transitions between the two states. We here address the accretion geometry in the intermediate state. (Physically different is the so-called very high/intermediate spectral state (discussion by Fender et al. 2004, model of disk fragmentation Meyer 2004).)

After a short description of the disk corona model in Sect. 2 we analyze in Sect. 3 the physics of an ADAF above a disk. One of the key questions is whether heat can be drained from the upper ADAF and radiated away. We investigate the processes in the two-temperature regime which extends over most of the vertical height, as well as those in the thin layer above the disk surface where ion and electron temperature couple. In Sect. 4 we derive under which conditions condensation of matter from the ADAF into the disk occurs, the necessary process to allow an inner disk to survive. In Sect. 5 we discuss how this picture can be related to the observed intermediate states.

\section{Accretion geometry - spectral states}

The key feature to understand the accretion geometry in the hard state is the evaporation of gas from the disk, feeding the hot flow. The disk corona model (Meyer et al. 2000a) describes the interaction between the hot corona and the cool disk below via energy and mass exchange. Numerical calculations (Meyer et al. 2000a; Liu et al. 2002) show that the evaporation rate reaches a maximum of about 1 percent of the Eddington accretion rate at a distance of several hundred Schwarzschild radii. Such a character, the maximum, provides a physical explanation for the occurrence of hard and soft states in X-ray binaries and the transitions between them (Meyer et al. 2000b): If the mass flow in the disk is below this maximum value, as usual in quiescence, the disk is truncated at the distance where all matter is evaporated, leaving inside a pure coronal flow/ADAF, which produces the hard spectrum. If the mass flow increases as during rise to an outburst the edge of the disk moves inward. If the mass flow in the disk becomes higher than the maximal evaporation rate, the disk can extend down to the last stable orbit and the spectrum 


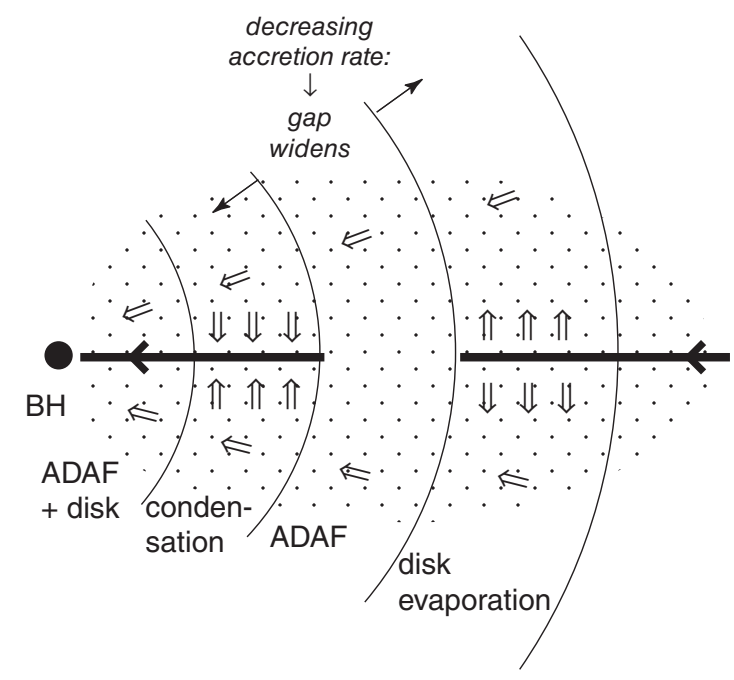

Fig. 1. Schematic drawing of the accretion flow in disk and corona, a gap formed due to evaporation.

is soft. During outburst decline the opposite happens, the inner edge of the disk retreats, in agreement with the observations.

In spite of this clear distinction of hard and soft states in the standard picture a decrease of the mass flow rate can cause a more complicated accretion geometry, as a gap in the disk at that distance where the evaporation efficiency is maximal (Fig. 1). Then the inner disk is affected by accretion towards the center and evaporation of matter diffusing outwards. The question is: can despite these processes a weak interior disk exist during the hard, ADAF-dominated state?

\section{Physics of an ADAF above a disk}

\subsection{Consequences of thermal conduction}

In a pure ADAF, almost all the accretion energy is stored in ions. Therefore, the ion temperature is near virial, the flow has a high vertical extension, the density is low (review Narayan et al. 1998). The coupling between electrons and ions is very poor and radiation is quite weak. The situation is different if there is a disk below the ADAF. Due to the large temperature difference between hot gas and cool disk, thermal conduction results in cooling of electrons. While the ion temperature is not much affected the electron temperature drops with height until at some $T_{\mathrm{e}}=T_{\mathrm{cpl}}$ coupling between ions and electrons becomes efficient and from then on ion and electron temperatures are the same, $T_{\mathrm{i}}=T_{\mathrm{e}}$. This constitutes a huge drop in ion temperature and causes a large increase in density as the pressure in the vertical extent keeps almost constant. As a consequence, Bremsstrahlung becomes much more important than in a typical ADAF. If the pressure exerted by the ADAF is large enough, Bremsstrahlung can be so efficient that all the heat drained from the ADAF by thermal conduction is radiated away in this layer. As in the standard disk corona model, this decides on evaporation and condensation of gas from and to the disk.

\subsection{Properties of the hot flow above a disk}

For our analysis we use the solutions of self-similar advectiondominated flows by Narayan \& Yi (1995). The properties of these flows scale with black hole mass, mass flow rate and distance from the black hole. They further depend on the viscosity and the assumed magnetic field strength. Following Narayan et al. (1998) the magnetic pressure is written as

$p_{\mathrm{m}}=(1-\beta) \rho c_{\mathrm{s}}^{2}$

with $\beta$ ratio of gas pressure to total pressure, $\rho$ density and $c_{\mathrm{s}}$ isothermal sound speed. Recent shearing box simulations of turbulence driven by the magnetorotational instability in a collisionless plasma by Sharma et al. (2006) yield $\beta$ values around 0.8 . We take this value for our analysis. As ratio of specific heats of the magnetized plasma we take $\gamma=(8-3 \beta) /(6-3 \beta)$ (Esin 1997) though the true value would require a more detailed analysis.

For the chemical abundance a hydrogen mass fraction of 0.75 was used. The solutions for pressure, electron number density, viscous dissipation of energy per unit volume $q^{+}$and isothermal sound speed are

$p=1.87 \times 10^{16} \alpha^{-1} m^{-1} \dot{m} r^{-5 / 2} \mathrm{~g} \mathrm{~cm}^{-1} \mathrm{~s}^{-2}$,

$n_{\mathrm{e}}=5.91 \times 10^{19} \alpha^{-1} m^{-1} \dot{m} r^{-3 / 2} \mathrm{~cm}^{-3}$

$q^{+}=2.24 \times 10^{20} \mathrm{~m}^{-2} \dot{m} r^{-4} \mathrm{erg} \mathrm{cm}^{-3} \mathrm{~s}^{-1}$,

$c_{\mathrm{s}}^{2}=1.67 \times 10^{20} \mathrm{r}^{-1} \mathrm{~cm}^{2} \mathrm{~s}^{-2}$,

where $\alpha$ is the viscous coefficient, $m$ the black hole mass in units of solar mass $M_{\odot}, \dot{m}$ the mass flow rate in units of Eddington accretion rate $\dot{M}_{\text {Edd }}=1.39 \times 10^{18} \mathrm{~m} \mathrm{~g} / \mathrm{s}$, and $r$ the radius in units of Schwarzschild radius $R_{\mathrm{S}}=2.95 \times 10^{5} \mathrm{~m} \mathrm{~cm}$. The ion number density is $n_{\mathrm{i}}=n_{\mathrm{e}} / 1.077$. In an ADAF ion and electron temperatures $T_{\mathrm{i}}$ and $T_{\mathrm{e}}$ closely follow

$T_{\mathrm{i}}+1.077 T_{\mathrm{e}}=1.98 \times 10^{12} r^{-1} \mathrm{~K}$

and, if $T_{\mathrm{e}}$ is much smaller than $T_{\mathrm{i}}$ this value can be taken for $T_{\mathrm{i}}$ alone. Besides viscous heating $q^{+}$compressive heating $q^{\mathrm{c}}=\frac{1}{(1-\beta)} q^{+}$(Esin 1997) is important. From the scaled values we determine the vertical conductive heat flux $F_{\mathrm{c}}$ and the temperature at which ions and electrons couple.

The electron temperature has practically no influence on the dynamics of the flow and the thermodynamics of the ion gas and is subrelativistic, $k T_{\mathrm{e}}<m_{\mathrm{e}} c^{2}$ ( $k$ Boltzmann constant, $m_{\mathrm{e}}$ electron mass, $c$ speed of light). The energy transfer from ions to electrons is given by Stepney (1983). Since in the two-temperature advection-dominated hot flow the ions generally are at a much higher temperature than the electrons a simplified formula (Liu et al. 2002) can be used

$$
\begin{aligned}
q_{\mathrm{ie}} & =3.59 \times 10^{-32} \mathrm{~g} \mathrm{~cm}^{5} \mathrm{~s}^{-3} \mathrm{deg}^{-1} n_{\mathrm{e}} n_{\mathrm{i}} T_{\mathrm{i}}\left(\frac{k T_{\mathrm{e}}}{m_{\mathrm{e}} c^{2}}\right)^{-\frac{3}{2}} \\
& =1.05 \times 10^{35} \mathrm{~g} \mathrm{~cm}^{-1} \mathrm{~s}^{-3} \mathrm{deg}^{3 / 2} T_{\mathrm{e}}^{-3 / 2} \alpha^{-2} m^{-2} \dot{m}^{2} r^{-4} .
\end{aligned}
$$

Cooling of electrons occurs through a variety of channels. Bremsstrahlung and synchrotron (-Compton) radiation (depending on the strength of the magnetic field) limit the electron temperature in the inner ADAF region. The new element is that an underlying disk drains heat. This is a new cooling process that competitively limits the peak electron temperature. This process determines the drop of electron temperature with height $z$ above the disk. The downward heat flux increases from $F_{\mathrm{c}}=0$ at large height to a saturation value at the bottom where ion and electron temperatures finally couple, at $z=z_{\text {cpl }}$. For our approximation we take $z_{\mathrm{cpl}}$ as small compared to the extent of the ADAF (in our example of order 10\%). The heat flux follows from the two relations

$F_{\mathrm{c}}=-\kappa_{0} T_{\mathrm{e}}^{5 / 2} \mathrm{~d} T_{\mathrm{e}} / \mathrm{d} z$

$\frac{\mathrm{d} F_{\mathrm{c}}}{\mathrm{d} z}=-q_{\mathrm{ie}}\left(T_{\mathrm{e}}\right)$ 
by integration over this temperature interval. We get

$F_{\mathrm{c}}{ }^{2}=\kappa_{0}\left(K n_{\mathrm{i}} n_{\mathrm{e}} T_{\mathrm{i}}\right)\left(T_{\mathrm{m}}{ }^{2}-T_{\mathrm{e}}{ }^{2}\right)$

with $K=1.64 \times 10^{-17} \mathrm{~g} \mathrm{~cm}^{5} \mathrm{~s}^{-3} \mathrm{deg}^{1 / 2}$. The integration of $z$ over $T_{\mathrm{e}}$ (Eq. (5)) gives the relation between the maximal electron temperature $T_{\mathrm{m}}$ and $z_{\mathrm{m}}$, the height at which $T_{\mathrm{m}}$ is reached. This yields

$T_{\mathrm{m}}^{5 / 2}=1.39 z_{\mathrm{m}}\left(K n_{\mathrm{i}} n_{\mathrm{e}} T_{\mathrm{i}} / \kappa_{0}\right)^{1 / 2}$.

For the height $z_{\mathrm{m}}$ we take the vertical scaleheight for $n_{\mathrm{e}} n_{\mathrm{i}}$ which is $1 / \sqrt{2}$ of the density scaleheight $c_{\mathrm{s}} / \Omega_{\mathrm{K}}\left(\Omega_{\mathrm{K}}\right.$ Kepler angular velocity) of Narayan et al. (1998). The value of $T_{\mathrm{m}}$ finally yields the heat flux near the bottom $\left(z=z_{\mathrm{cpl}}\right)$ as

$F_{\mathrm{c}}^{\mathrm{ADAF}}=-\kappa_{0}^{3 / 10}\left(K n_{\mathrm{i}} n_{\mathrm{e}} T_{\mathrm{i}}\right)^{7 / 10}\left(1.39 z_{\mathrm{m}}\right)^{2 / 5}$.

\subsection{Coupling of ion and electron temperature}

As pointed out in Sect. 3.1 only if the electron temperature has dropped sufficiently ions and electrons start to couple. This requires that the heat transfer from ions to electrons balances viscous and compressive heating, $q_{\mathrm{ie}}=q^{+}+q^{\mathrm{c}}$. The coupling temperature follows from Eqs. (2) and (4). Since the coupling occurs at very low height the density there is higher than the height averaged value (Eq. (2)), by a factor is $2 / \sqrt{\pi}$ (vertical density distribution $n=n_{0} \exp \left(-z^{2} / H^{2}\right)$. This yields the coupling temperature as

$T_{\text {cpl }}=1.98 \times 10^{9} \alpha^{-4 / 3} \dot{m}^{2 / 3}=1.24 \times 10^{9} \mathrm{~K}$,

the numerical value for $\alpha=0.2$ and $\dot{m}=0.02$ (see Sect. 4). The sudden decrease of ion temperature leads to a sudden increase of ion and electron density, and the electrons radiate efficiently.

\subsection{The radiating layer close to the disk surface}

The parameters of the ADAF determine what happens in the layer between coupling and disk surface. Different regimes are possible, depending on the pressure in the ADAF. Case (1): if the pressure is high, the density in the lower layer also is high. Then the conductive flux drained from the ADAF is already efficiently radiated away at some height before the disk surface is reached. Radiative cooling must be additionally balanced by heat released from gas condensing into the disk. Case (2): if the pressure is low, only part of the conductive flux can be radiated away, the remaining part will heat up cool disk matter, i.e. matter evaporates from the disk to the corona. A limiting case in between, case (3), occurs if the ADAF pressure allows the thermal heat flux drained from the ADAF to be radiated away exactly at the disk surface. Then there is neither condensation nor evaporation, no mass exchange between the disk and the ADAF.

\section{Condensation of gas from the ADAF into the inner disk?}

From the energy balance in the radiating layer we derive the condition for the different cases. We use here a simplified form of the energy equation, keeping only the dominant contribution of internal heat, pressure work, and thermal conduction, together with Bremsstrahlung cooling $n_{\mathrm{e}} n_{\mathrm{i}} \Lambda(T)$

$\frac{\mathrm{d}}{\mathrm{d} z}\left[\dot{m}_{z} \frac{\gamma}{\gamma-1} \frac{\mathfrak{R} T}{\mu}+F_{\mathrm{c}}\right]=-n_{\mathrm{e}} n_{\mathrm{i}} \Lambda(T)$, with $\mathfrak{R}$ gas constant, $\mu$ molecular weight, $\dot{m}_{z}$ vertical mass flow rate between ADAF and disk per unit area. We express density by temperature and (constant) gas pressure $\beta p$. This value is taken at the bottom of the ADAF region, and, as for the density, is slightly higher than the vertical mean pressure (Eq. (2)). Assuming free-free radiation for $T_{\mathrm{e}} \geq 10^{7.5} \mathrm{~K}, n_{\mathrm{e}} n_{\mathrm{i}} \Lambda(T)$ becomes $\frac{0.25}{k^{2}}(\beta p)^{2} b T^{-3 / 2}$ with $b=10^{-26.56} \mathrm{~g} \mathrm{~cm}^{5} \mathrm{~s}^{-3} \mathrm{deg}^{-1 / 2}$ (Sutherland \& Dopita 1993). For formal simplicity we use this law also for smaller $T$. The justification is that because of the very steep temperature profile below $10^{7.5} \mathrm{~K}$ such regions contribute only a negligible amount to cooling of this layer (Liu et al. 1995). Contributions of gravitational energy release, frictional heating, and side-wise advection of mass and energy can be neglected for small extent of this layer. Likewise kinetic energy is negligible since at the high density the flow is highly subsonic.

To solve the second-order differential equation Eq. (11), we change to $T$ as independent variable and define a new variable $g(T) \equiv \kappa_{0} T^{3 / 2} \mathrm{~d} T / \mathrm{d} z=-F_{\mathrm{c}} / T$. We now get the first-order differential equation

$$
\begin{aligned}
g \frac{\mathrm{d} g}{\mathrm{~d} \ln T} & =\frac{0.25 \beta^{2} p^{2}}{k^{2}} \kappa_{0} b+\dot{m}_{z} \frac{\gamma}{\gamma-1} \frac{\mathfrak{R}}{\mu} g-g^{2} \\
& =-\left(g-g_{1}\right)\left(g-g_{2}\right),
\end{aligned}
$$

with

$g_{1}=\frac{\dot{m}_{z}}{2} \frac{\gamma}{\gamma-1} \frac{\mathfrak{R}}{\mu}+\sqrt{\left(\frac{\dot{m}_{z}}{2} \frac{\gamma}{\gamma-1} \frac{\mathfrak{R}}{\mu}\right)^{2}+\frac{0.25 \beta^{2} p^{2}}{k^{2}} \kappa_{0} b}$

and $g_{2}$ differing from $g_{1}$ only by the sign of the square root.

This equation has to be solved with the upper boundary at $T=T_{\text {cpl }}$, where $F_{\text {c }}^{\mathrm{ADAF}}$ is the heat flux coming down from to the two-temperature ADAF region, and the lower boundary at $T=0$, the disk surface, with $F_{\mathrm{c}}=0 . \dot{m}_{z}$ is the Eigenvalue. The only solution that fulfills both boundary conditions is the singular solution $g(T)=g_{1}$. We get

$$
\begin{aligned}
& \dot{m}_{z}=\frac{\gamma-1}{\gamma} \frac{-F_{\mathrm{c}}^{\mathrm{ADAF}}}{\mathfrak{R} T_{\mathrm{cpl}} / \mu}[1-C], \\
& C=\kappa_{0} b\left(\frac{0.25 \beta^{2} p^{2}}{k^{2}}\right)\left(\frac{T_{\mathrm{cpl}}}{F_{\mathrm{c}}^{\mathrm{ADAF}}}\right)^{2} .
\end{aligned}
$$

The sign of the square bracket decides on evaporation or condensation: if $C$ is less than 1, the radiation losses are too weak, $\dot{m}_{z}$ is positive and the heat flow is used up to heat the evaporating gas to the boundary temperature $T_{\mathrm{cpl}}$. If, on the other hand, $C>1$, then the gas in this layer is efficiently cooled, sinks down and condenses. Note that at the coupling boundary for a rising flow additional heat is used up to heat the ions to the ADAF value $T_{\mathrm{i}}$. This diminishes $F_{\mathrm{c}}^{\mathrm{ADAF}}$ in Eq. (14). The opposite holds for a condensing flow. This limits the use of Eq. (14) to the neighborhood of $C=1$, the borderline case.

The value of $C$ scales with $\alpha, \dot{m}$ and $r$, but not with $m$. This shows that the same situation holds for supermassive and stellar black hole accretion. It depends on the gas pressure fraction $\beta$. For $\beta=0.8$ we get

$C=0.96 \alpha^{-28 / 15} \dot{m}^{8 / 15} r^{-1 / 5}$.

We take $\alpha=0.2$ (corresponding to $\alpha=0.3$ in Shakura-Sunyaev notation). The intermediate states appear in connection with the spectral state transitions. Maccarone (2003) found from a detailed investigation of these transitions in X-ray binaries $\dot{m}=0.02$. With these values $C$ becomes 1 at the distance of 
about 80 Schwarzschild radii. Note significant dependence on the value of $\beta$. The important outcome is the dependence of the value $C$ on $\dot{m}$ and $r$, which allows to derive the conditions for the existence of cool matter below the ADAF.

\section{Understanding the intermediate state}

The dependence of $C$ on mass flow rate and distance yields the following picture. If during outburst decline the mass flow rate in the disk decreases to a value equal to the maximal evaporation rate the disk breaks up where the evaporation is most efficient. A gap forms, inside a cool disk still exists, an ADAF above it. Only if condensation is possible this disk can survive. Our analysis predicts: If the mass flow rate declines slowly, from the transition rate to the minimal rate for condensation to occur ( $\dot{m} \approx 0.006, C=1$ at the last stable orbit), an intermediate spectral state appears, caused by a sustained inner disk underneath an ADAF. Note that due to the low recondensation rate a soft contribution to the spectrum is weak. If the rate drops quickly, almost no condensation occurs, the disk disappears as a consequence of accretion and evaporation without new mass supply from the ADAF.

Kalemci et al. (2004) studied intermediate states of several $\mathrm{X}$-ray transients. Our analysis suggests that these states are related to a phase where the disk breaks up, but inner cool matter remains by recondensation from an ADAF that has formed. But comparison with observations still requires further analysis, in particular of processes which tend to limit the inward extent of the remaining cool matter.

During rise to outburst the situation is different, the inner edge of the disk moves inward within a viscous time, inside only an ADAF.

If the mass flow rate in the disk is always well below the maximal evaporation rate the disk is truncated all the time, no condensation occurs, since no cool matter exists inside the truncation, no thermal conduction can appear. This picture is supported by observations of XTE J1118+480, a system which stays in the hard state even during outburst (Esin et al. 2001).

\section{Conclusions}

We introduce a new feature of accretion flows onto black holes, the advection-dominated accretion flow affected by thermal conduction to a cool disk underneath. Our analysis shows that in the inner region condensation of matter from the hot flow into the disk is possible. This allows the existence of cool matter together with an already formed ADAF as indicated by the observed reflection and $\mathrm{Fe} \mathrm{K} \alpha$ line in the intermediate spectral state.

Acknowledgements. The authors acknowledge support by the National Natural Science Foundation of China (NSF-10533050) and the BaiRenJiHua program of the Chinese Academy of Sciences.

\section{References}

Esin, A. A. 1997, ApJ, 482, 400

Esin, A. A., McClintock, J. E., \& Narayan, R. 1997, ApJ, 489, 865 Esin, A. A., McClintock, J. E., Drake, J. J., et al. 2001, ApJ, 555, 483 Fender, R. P., Belloni, T. M., \& Gallo, E. 2004, MNRAS, 355, 1105 Kalemci, E., Tomsick, J. A., Rothschild, R. E., et al. 2003, ApJ, 603, 23 Liu, F. K., Meyer, F., \& Meyer-Hofmeister, E. 1995, A\&A, 300, 823 Liu, B. F., Mineshige, S., Meyer, F., et al. 2002, ApJ, 575, 117 Maccarone, T. J. 2003, A\&A, 409, 697

McClintock, J. E., \& Remillard, R. A. 2006, in Compact Stellar X-ray Sources, ed. W. H. G. Lewin, \& M. van der Klis (Cambridge Univ. Press) Meyer, F. 2004, Progress of Theoretical Physics Suppl., 155, 45 Meyer, F., Liu, B. F., \& Meyer-Hofmeister, E. 2000a, A\&A, 361, 175

Meyer, F., Liu, B. F., \& Meyer-Hofmeister, E. 2000b, A\&A, 354, L67 Narayan, R., Mahadevan, R., \& Quataert, E. 1998, in The Theory of Black Hole Accretion Discs, ed. M. A. Abramowicz et al. (Cambridge Univ. Press), 48 Narayan, R., \& Yi, I. 1995, ApJ, 452, 710

Sharma, P., Hammet, W., Quataert, E., et al. 2006, ApJ, 637, 952

Stepney, S. 1983, MNRAS, 202, 467

Sutherland, R. S., \& Dopita, M. A. 1993, ApJS, 88, 253

Zycki, P. T., Done, C., \& Smith, D. A. 1998, ApJ, 496, L25 